\title{
On the Mass and on the Dynamical Properties of the Sexaquark
}

\section{Franco Buccella*}

INFN, Sezione di Napoli, Italy

E-mail: buccelladromal, infn.it

The proposal of a bound state $u u d d s s$ as a candidate for the dark mass encourages the research on the properties of such a state expected in the framework of QCD .

To this extent we try to get an approximate evaluation of the mass of that particle in terms of the chromomagnetic and chromoelectric interactions between the constituents, which is applied to the lightest ordinary hadrons and the two nonets of tetraquarks.

The way in which the constituents combine to form the singlet under color, spin and flavour, gives an useful information on the dynamical behaviour and the stability of the uuddss particle.

Corfu Summer Institute 2019 "School and Workshops on Elementary Particle Physics and Gravity" (CORFU2019)

31 August - 25 September 2019

Corfu, Greece

${ }^{*}$ Speaker. 


\section{Introduction}

It has been proposed [W] that, after the baryogenesis, at a temperature of about $150 \mathrm{Mev}$, when the presence of strange quarks is of the order, of course smaller, than for the light ( $u$ and $d$ ) quarks, instead of forming hyperons, $\Lambda, \Sigma, \Xi$, for the most part they combine to form states of 6 quarks, uuddss, with mass smaller than twice the mass of the $\Lambda$ particle. If their mass is in the narrow range $1860-1880 \mathrm{MeV}$ they should be stable and produced more than a pair of baryons with $S=-2$ and, if their number is not reduced up to the present times, should be a candidate for the dark matter . With respect to this hypothesis it has been stressed [ [ ] that even with the mass in that range these particles, the sexaquarks, should almost disappear in a way dependent by the rapidity of the inelastic reactions induced by $K^{+}$or $\pi^{+}$.

The presence of sexaquarks may account for the minor primordial production of $L i^{7}$ [目] .

The ingenious [ [ $⿴$ ] proposal that these particles should consist of three scalar diquarks $(u d, u s, d s)$ transforming as a $\overline{3}$ of color and flavor combined, as good bosons, antisymmetrically in color and flavor has lead to a prediction of a small mass around $1400 \mathrm{MeV}$, which would imply the instability of nuclei .

Here first on the basis of Pauli principle by the comparison with the other hadrons with constituents in S-wave, for which the chromomagnetic interaction describes successfully the spectrum, we expect for the sexaquark a mass smaller than twice the mass of the $\Lambda$. Then, by assuming as in [四] that the sexaquark is built as the antisymmetric combination in color and flavor of three scalar diquarks transforming as a $\overline{3}$ with respect to both $S U(3)$, we evaluate its mass, which comes out $1883 \mathrm{MeV}$ just above the range required for its stability .

The strong difference with respect to [ [ 7 ] is the different interpretation of the lightest nonet tetraquark : in [䧃] it is supposed to be built by scalar diquarks, transforming as a $\overline{3}$ of color and flavor, and their antiparticles, while we assume that it is the lower eigenstate of the chromomagnetic interaction in the two-dimensional space of the scalar nonets, which allows to explain the very large couplings of the states of the lighter nonet to the channels with two pseudoscalar mesons, while the higher eigenstate built with light constituents, the $f^{0}(1370)$, is predicted to decay mainly into four pions in agreement with experiment [5] .

We derive the mass of the sexaquark from the study of the masses of the $\left(\frac{1}{2}\right)^{+}$and $\left(\frac{3}{2}\right)^{+}$or of the two lowest scalar tetraquark nonets.

In terms of the masses of the diquarks we find for the sexaquark a mass of about $1883 \mathrm{MeV}$.

The way, in which the three sexaquarks combine to build the sexaquark have important consequences on its dynamical properties with respect both to its strong and weak interactions .

In fact the interactions, which transform the $s$ quark into a $u$ or a $d$ are forbidden by the asymmetry in flavor of its $S U(3)$ wave function; if they do not change the spin of the tetraquark, also by the spin-statistics theorem .

In the following section we describe a constituent model, where QCD plays the main role with its chromomagnetic and chromoelectric interactions, reproducing the spectrum with all the constituents in S-wave, the baryons, the mesons and the tetraquark scalar nonets . In the third section we first evaluate the order of magnitude of the mass of the sexaquark through an analogy with baryons. Then we evaluate its mass, assuming as in [团] that it is built with scalar diquarks, transforming as a $\overline{3}$ of color and flavor. 
In the fourth section we stress that the stability properties, which are a consequence of the structure assumed for the sexaquark, and the difficulty to transform it into a pair of $\Lambda$ 's or two baryons. Finally we shall give our conclusions .

\section{Hadron spectrum from QCD}

After the proposal that QCD is the theory of strong interactions [8]], De Rujula, Georgi and Glashow [9] realized that the fine structure (the chromomagnetic interaction, CMI) accounts for the mass differences between $\Delta$ and the nucleon and between $\Sigma$ and the $\Lambda$. In this framework, there is the prediction :

$$
M\left(\Xi^{*}\right)-M(\Xi)=M\left(Y^{*}\right)-M(\Sigma)
$$

which had been previously obtained, by assuming the same coefficients for the mass terms transforming both as an octet for the decuplet and the octet of baryons.

Applying the same approach to the charmed baryons $\Sigma_{c}$ and $\Lambda_{c}$, they predicted a mass difference high enough to allow the strong decay $\Sigma_{c}^{+} \rightarrow \Lambda_{c}+\pi^{+}$, in agreement with the discovery of both particles in a neutrino experiment [ए]]. The chromomagnetic interaction between the three constituent quarks gives a contribution to the mass of the baryon, they form, which depends on the quadratic Casimir of $S U(6)$ color spin, $S U(6)_{c s}, S U(3)$ color, $S U(3)_{c}$ and $S U(2)$ spin, $S U(2)_{s}$, and is proportional with a negative factor to [ए]] [ए]] :

$$
C_{6}(q q q)-\frac{C_{3}(q q q)}{2}-\frac{C_{2}(q q q)}{3}-6
$$

Since the color singlets built with three quarks with spin parity $\left(\frac{1}{2}\right)^{+}$and $\left(\frac{3}{2}\right)^{+}$belong to the 70 and 20 with Casimir $\frac{33}{4}$ and $\frac{21}{4}$, respectively, the proportionality factor is :

$$
\frac{M_{N}-M_{\Delta}}{4}=-73.25 \mathrm{MeV}
$$

The masses of $N$ and $\Delta$ may be obtained by adding the sum of the effective masses of the constituents, which is given by $\frac{M_{N}+M_{\Delta}}{2}$, since the chromagnetic contributions to their masses are opposite.

The mass difference between the $\Sigma$ and $\Lambda$ hyperons is a consequence of the different girochromomagnetic factors of the light ( $\mathrm{u}$ and $\mathrm{d}$ ) and strange quark (s). Indeed the total spin of the two light quarks in the $\Lambda$ is 0 and therefore the chromomagnetic contribution to the mass of that particle is equal to the one for the nucleon. Therefore the mass difference between the strange and the light constituents of the baryons is just given by the difference $M_{\Lambda}-M_{N}$. Instead for the $\Sigma$ the total spin of the two light quarks is 1 , which implies a total chromomagnetic contribution to its mass $\left[\frac{1}{6}-\frac{2 k_{s}}{3}\right]\left(M_{\Delta}-M_{N}\right)$, where $k_{s}=0.6$ is the ratio of the strange and light girochromomagnetic factors .

As long as for the charmed baryons the chromomagnetic interaction between a light and a charmed quark is weaker, mainly for the smaller gyro-chromomagnetic factor of the charmed quark inversely proportional to its mass. To reproduce the masses a factor $k_{1 c}=0.24$ is needed for the CMI and an effective mass for the charmed quark of $1715 \mathrm{MeV}$.

The $\Sigma_{b}$ and $\Lambda_{b}$ particles have a mass difference even larger, as expected. 
For the mesons $\left(\pi, K \rho, K^{*}\right)$ the chromomagnetic contribution is proportional with a positive coefficient to

$$
C_{6}(q \bar{q})-\frac{C_{3}(q \bar{q})}{2}-\frac{C_{2}(q \bar{q})}{3}-4
$$

The vector and the pseudoscalar mesons belong to the 35 and to the 1 representations of $S U(6)_{c s}$ with quadratic Casimir 6 and 0, respectively, and therefore the proportionality coefficient for the mesons built with light constituents is :

$$
\frac{3\left(M_{\rho}-M_{\pi}\right)}{16}=119 \mathrm{MeV}
$$

Interestingly enough, both the sign and the order of magnitude of the mass differences appearing in Eq. (2.3]) and Eq. (2.5) have been obtained in [[13], as a consequence of the sum rules proposed in [ㄴ]], in the framework of the transformation, which relates constituent and current quarks [ㄷ]] [ए6] .

The chromomagnetic contribution for the strange pseudoscalar is proportional to $k_{s}$ and one expects

$$
\frac{M_{K^{*}}-M_{K}}{M_{\rho}-M_{\pi}}=k_{s}^{\prime}=0.62
$$

The sum of the light constituent masses in the mesons is given by $\frac{3 M_{\rho}+M_{\pi}}{4}=2 m_{q}^{\prime}=712 \mathrm{MeV}$, while for the strange mesons is given by $\frac{3 M_{K^{*}}+M_{K}}{4}=m_{q}^{\prime}+m_{s}^{\prime}=793 \mathrm{MeV}$, implying $m_{s}^{\prime}=487 \mathrm{MeV}$ and for the ratio $\frac{m_{q}^{\prime}}{m_{s}^{\prime}}=0.63$.

So one needs a larger chromomagnetic interaction and a smaller effective masses for the light and the strange quarks than in the case of the baryons. Both these properties can be understood by the more intense chromoelectric attraction between a quark and an antiquark, which form a color singlet with respect to two quarks, which combine in a $\overline{3}$ of SU(3) color. Indeed, the stronger attraction implies a smaller constituent mass and a larger contact interaction. In fact, for the charmed mesons $D$ and $D^{*}$, a slightly smaller mass, $1615 \mathrm{MeV}$, and larger $k_{2 c}=0.26$ are needed with respect to the charmed baryons. Also, the values found for the $c \bar{c}$ states, $1535 \mathrm{MeV}$ for the mass of the charmed quark and $K_{c}^{2}=0.186$ for the square of the gyrochromomagnetic factor can be understood as a consequence of the smaller distance between the constituents.

The mass of $3621.40 \mathrm{MeV}$ of the $\Xi_{c c}^{++}$recently found by $L H C b$ [ [ $]$ implies an effective mass of the constituent charmed quarks of $1665 \mathrm{MeV}$, somewhat larger than the one found for the charmed mesons and $\Lambda_{c}$.

In conclusion we have the following expressions for the masses of the particles previously mentioned:

$$
\begin{gathered}
M_{N}=1086-146.5=939.5 \mathrm{MeV}[939] \\
M_{\Lambda}=1263-146.5=1116.5 \mathrm{MeV}[1116] \\
M_{\Sigma}=1263-146.5\left(0.8-\frac{1}{3}\right)=1194 \mathrm{MeV}[1193] \\
M_{\Xi}=1440-146(0.8-0.13)=1342 \mathrm{MeV}[1318] \\
M_{\Delta}=1086+146.5=1232.5 \mathrm{MeV}[1232]
\end{gathered}
$$




$$
\begin{gathered}
M_{Y^{*}} 1263+146.5\left(0.4+\frac{1}{3}\right)=1370 \mathrm{MeV}[1385] \\
M_{\Xi^{*}}=1440+146.5(0.4+0.12)=1516 \mathrm{MeV}[1530] \\
M_{\Omega}=1617+146(0.36)=1670 \mathrm{MeV}[1672] \\
M_{\pi}=612-476=135 \mathrm{Mev}[135] \\
M_{K}=793-476 \times 0.62=498 \mathrm{MeV}[496] \\
M_{\rho}=612+119 \times \frac{4}{3}=771 \mathrm{MeV}[770] \\
M_{K^{*}}=793+119 \frac{4}{3} \times 0.62=891 \mathrm{MeV}[892] \\
M_{\Phi}=974+119 \times \frac{4}{3} \times(0.62)^{2}=1036 \mathrm{MeV}[1020]
\end{gathered}
$$

where we have written the experimental values in square brakets and the larger deviation is for $\Xi$ as is the case for the Gell-Mann Okubo formula [ []] :

$$
M_{\Xi}=\frac{3 M_{\Lambda}+M_{\Sigma}-2 M_{N}}{2}=1333 \mathrm{MeV}
$$

It is instructive to compare the description of the spectrum of the $\left(\frac{1}{2}\right)^{+}$and $\left(\frac{3}{2}\right)^{+}$baryons just described by a $S U(3)$ analysis assuming isospin invariance from the tensor products :

$$
\begin{gathered}
8 \times 8=1+8_{A}+8_{S}+10+\overline{10}+27 \\
10 \times \overline{10}=1+8+27+64
\end{gathered}
$$

with all the representations in the r. h. s. of eqs.(21) and (22), except the 10 and the $\overline{10}$, containing an isospin singlet, we can costruct the following combinations of the masses of the octet and of the decimet, which receives contributions from the different representation :

$$
\begin{gathered}
M_{1}=\frac{2 M_{N}+M_{\Lambda}+3 M_{\Sigma}+2 M_{\Xi}}{8}=2 m+m_{s}-146.5 \mathrm{MeV}\left[1-\frac{\left(1-k_{s}\right)\left(6-\frac{2 k_{s}}{3}\right)}{8}\right]=1157.5(1163.6) \mathrm{MeV} \\
M_{8_{A}}=\frac{M_{\Xi}-M_{N}}{2}=m_{s}-m-146.5 \mathrm{MeV}\left(1-k_{s}\right)\left(1-\frac{k_{s}}{3}\right)=200.5(189.5) \mathrm{MeV} \\
M_{8_{S}}=\frac{3 M_{\Sigma}-M_{N}-M_{\Lambda}-M_{\Xi}}{3}=146.5 \mathrm{MeV}\left(1-k_{s}\right)\left(1+\frac{k_{s}}{9}\right)=62.5(68.7) \mathrm{MeV} \\
M_{27}=\frac{3 M_{\Lambda}+M_{\Sigma}-2 M_{N}-2 M_{\Xi}}{4}=-146.5 \mathrm{MeV} \frac{\left(1-k_{s}\right)^{2}}{6}=-3.9(6.75) \mathrm{MeV} \\
M_{1}^{\prime}=\frac{M_{\Omega}+2 M_{\Xi^{*}}+3 M_{Y^{*}}+4 M_{\Delta}}{10}=2 m+m_{s}+146.5 \mathrm{MeV} \frac{3+2 k_{s}+\left(k_{s}\right)^{2}}{6}=1374(1378.5) \mathrm{MeV} \\
M_{8}^{\prime}=\frac{2 M_{\Omega}+2 M_{\Xi^{*}}-4 M_{\Delta}}{6}=m_{s}-m-146.5 \mathrm{MeV}\left(1-k_{s}\right)\left(0.4+\frac{0.8 k_{s}}{3}\right)=133.5(135) \mathrm{MeV}
\end{gathered}
$$




$$
\begin{gathered}
M_{27}^{\prime}=\frac{3 M_{\Omega}-2 M_{\Xi^{*}}-5 M Y^{*}+4 M \Delta}{7}=146.5 \mathrm{MeV} \frac{\left(1-k_{s}\right)^{2}}{3}=7.8(-6.4) \mathrm{MeV} \\
M_{64}^{\prime}=\frac{M_{\Omega}-3 M_{\Xi^{*}}+3 M_{Y^{*}}+M_{\Delta}}{4}=0(1) \mathrm{MeV}
\end{gathered}
$$

The r. h. s.'s of eqs.(24-31) have been obtained from the experimental values $M_{N}=939 \mathrm{MeV}$, $M_{\Lambda}=1116 \mathrm{MeV}, M_{\Sigma}=1193 \mathrm{MeV} M_{\Xi}=1318 \mathrm{MeV}, M_{\Delta}=1232 \mathrm{MeV}, M_{Y^{*}}=1385 \mathrm{MeV}, M_{(\Xi)^{*}}=$ $1530 \mathrm{MeV}$ and $M_{\Omega}=1672 \mathrm{MeV}$.

The Gell-Mann Okubo hypothesis of contributions only from the 1 and 8 representations would imply vanishing values for the r. h. s.'s of eqs.(26), (29) and (30). Indeed those values are smaller than the others, in particular the last one, supporting the idea that they correspond to higher order of a perturbation transforming as an octet (in fact to construct a 64 of $S U(3)_{f}$ one needs the tensor product of three 8's). Since the approach here described predicts the remaining masses from the ones of the nucleon, the $\Lambda$, the $\Sigma$ and the $\Delta$ particles, the evaluation of the r. h. s.'s of eqs.(7-14) are given in terms of the four free parameters fixed by the three masses, $m_{q}=362 \mathrm{MeV}$ for the effective mass (including the chromoelectric actraction) of the light quarks, $m_{s}=539 \mathrm{MeV}$ for the strange one, $293 \mathrm{MeV}$ for the difference $M_{\Delta}-M_{N}$ coming for the chromomagnetic interaction and $k_{s}=0.6$ for the ratio between the girochromomagnetic factors of the strange and the light quarks to account for the difference $M_{\Sigma}-M_{\Lambda}$. We consider now the two nonets of scalar tetraquarks [ए]] [ए]], where the isoscalar states built with the light constituents are the $f^{0}(500)$ and $f^{0}(1370)$ resonances, the strange states are $k(700)$ and $K(1430)$, while the hidden strange ones are $f^{\prime}(980)$ and $a(980)$ or $f^{\prime}(1500)$ and $a(1450)$, respectively. For the states built with light costituents or only with one strange constituent there is no mixing between the $\overline{3} \times 3$ and the $6 \times \overline{6}$ states of $S U(3)_{f}$ . For the states with hidden strangeness the mixing is small, second order in $S U(3)_{f}$ breaking and will be neglected . Let us diagonalize the chromomagnetic interaction in the two-dimensional space of the states built with the $\mid(\overline{3}, 1, \overline{3}) \times 3,1,3)_{1,1}=\mid \alpha>$ and $\left.\mid(6,3, \overline{3}) \times \overline{6}, 3,3\right)_{1,1}=\mid \beta>$ of $S U(3)_{c} \times S U(2)_{s} \times S U(3)_{f}$. The matrix elements of the chromomagnetic interaction of these tetraquark states with no more than one strange costituent are proportional to :

$$
O=C_{6}(q q \bar{q} \bar{q})-2 C_{6}(q q)-2 C_{6}(\bar{q} \bar{q})+C_{3}(q q)+C_{3}(\bar{q} \bar{q})+\frac{2}{3} C_{2}(q q)+\frac{2}{3} C_{2}(\bar{q} \bar{q})(2.31)
$$

and the values $C_{6}(405)=14, C_{6}(21)=\frac{20}{3}$ and the well-known values of the $C_{3}$ and $C_{2}$ quadratic Casimir's imply :

$<\alpha|O| \alpha>=-4,<\beta|O| \alpha>=<\alpha|O| \beta>=-2 \sqrt{6}$, and $\left\langle\beta|O| \beta>=-\frac{22}{3}\right.$ with eigenvalues -0.49 and -10.84 , respectively.

If we take the same parameters found for the baryons the proportionality factor is $73.25 \mathrm{MeV}$ for the isoscalar states with light costituents and 73.25 multipled $\frac{1+k_{s}^{\prime \prime}}{2}$ for the strange states.

For the states with hidden strangness with isospin 0 and 1 one has to multiply $73.25 \mathrm{MeV}$ for the matrix elements :

$<$ h.s. $\alpha|O|$ h.s. $\alpha>=-4 k_{s}^{\prime \prime},<$ h.s. $\beta|O|$ h.s. $\alpha>=<$ h.s. $\alpha|O|$ h.s. $\beta>=-\sqrt{6} \frac{\left(1+k_{s}\right)^{2}}{2}$, and $<$ h.s. $\beta|O|$ h.s. $\beta>=$ $-\frac{2}{3} k_{s}^{\prime \prime}-\frac{5}{3}\left(1+k_{s}^{\prime \prime}\right)^{2}$. The small value of the less negative eigenvalue, -0.49 , implies for the sum of the costituent masses values slightly higher than the masses of the heavier nonet and the small differences between the masses imply that, at difference with what happens for the baryons and the 
mesons, the difference of the masses of the strange and the light quarks is small. We can obtain a good agreement with experiment for the masses of the heavier nonet and the two hidden strangness states of the lighter one with the following values of the parameters, $m^{\prime \prime}=351.5 \mathrm{MeV}, m_{s}^{\prime \prime}=403.5$ $\mathrm{MeV}$, the parameter proportional to the chromomagnetic contribution 73.25, as in the baryons, and $k_{s}^{\prime \prime}=0.62$. For the heavier nonet, we get the masses 1370, 1429 and $1501 \mathrm{Mev}$, while for the lighter nonet 612,811 and $980 \mathrm{MeV}$. For the first two we find values larger than the ones quoted now, but the determination of those values is affected by the large width of the two resonances. The singlet and the 405 of $S U(6)_{c s}$ are given by the following combinations :

$$
\begin{aligned}
& \mid 1>=\sqrt{\frac{1}{7}}(|\alpha>+\sqrt{6}| \beta>) \\
& \mid 405>=\sqrt{\frac{1}{7}}(\sqrt{6}|\alpha>-| \beta>)
\end{aligned}
$$

which implies that :

$$
\begin{aligned}
& \left|f^{0}(600)>=0.97\right| 1>+0.24 \mid 405> \\
& \left|f^{0}(1370)>=-0.24\right| 1>+0.97 \mid 405>
\end{aligned}
$$

Interestingly enough, this explains why the lowest one, which decays into two pions, has a very large width, while the other one decays mainly into four pions [5]. In fact, the $S U(6)_{c s}$ Casimir, which gives the most important chromomagnetic contribution to the masses, implies that the lighter state is almost a $S U(6)_{c s}$ singlet with an "open channel" [ㅁ] into two pions, which are also $S U(6)_{c s}$ singlets, while the heavier one transforms mainly as a 405 and therefore has an "open channel" into a pair of $\rho$ mesons, which transform as a 35 of $S U(6)_{c s}$ color spin [20] [2] . Also the states of the lighter nonet with one or two strange quarks have strong couplings to two pseudoscalars, while this is not the case for the heavier ones .

The small value of the mass found for the strange diquarks will bring to a small value for the mass of the sexaquark built by combining the three scalar diquarks transforming as the $\overline{3} \times \overline{3}$ of $S U(3)_{c} \times S U(3)_{f}$.

A general analysis of the spectrum of negative and positive pentaquarks built with the three lightest quarks can be found in [23] and the study of $3 q 3 \bar{q}$ hexaquarks in [24].

In conclusion we have a good description of the masses of the $\left(\frac{1}{2}\right)^{+}$and $\left(\frac{3}{2}\right)^{+}$baryons with effective masses (including the effect of the chromoelectric interaction) $m_{q}=362 \mathrm{MeV}, m_{s}=539 \mathrm{MeV}$, the coefficient of eq.(2) $73.25 \mathrm{MeV}$ and $k_{s}=0.60$ (to be compared with the ratio $\frac{m_{q}}{m_{s}}=0.66$ ), while for the mesons the masses of the $\pi, K, \rho, K^{*}$ and $\phi$ are well described with smaller values for the constituent masses, $m_{q}^{\prime}=306 \mathrm{MeV}, m_{s}^{\prime}=487.5 \mathrm{MeV}$, the value $119 \mathrm{MeV}$ for the coefficient of eq.(4), $k^{\prime}=0.62$ to be compared with the ratio $\frac{m_{q}^{\prime}}{m_{s}^{\prime}}=0.63$.

To reproduce the masses of the two tetraquark scalar nonets an effective mass of the strange quark in the scalar diquarks should be smaller than the one in the lower baryons and mesons. 


\section{Computation of the mass of the sexaquark}

Encouraged by the good description obtained for the masses of the lowest baryons and mesons and of the two scalar tetraquark nonets in our QCD inspired approach we try to evaluate the mass of the sexaquark, $\mathrm{S}$, in a similar way .

It is istructive to consider the cases, where the six quarks uuddss are combined in a singlet of $S U(3)_{c} \times S U(2)_{s} \times S U(3)_{f}$ with a completely antisymmetric wave function and where the sexaquark, $S$, is built by threee scalar diquarks, transforming as a $(\overline{3}, \overline{3})$ of $S U(3)_{c} \times S U(3)_{f}$ combined in an antisymmetric combination in color and flavor [䧃].

In the case of six light constituents uuuddd they may combine in two color singlets, uud, a proton, and $u d d$, a neutron, which together may build a deuteron. One may also consider a totally symmetric spatial wave function : an isospin singlet for the Pauli principle [22] implies that they transform as the 175 of $S U(6)_{c s}$ with quadratic Casimir $=12$.

Instead the uuddss $S U(3)_{f}$ singlets may combine into a pair $\Lambda \Lambda, \Sigma \Sigma$ or $N \Xi$, but also into a completely spatial symmetric wave function and in this case Pauli principle implies that a $S U(3)_{f}$ singlet (Young tableaux with three rows and two columns) combines with the 490 representation of $S U(6)_{c s}$ (Young tableaux with two rows and three columns) with quadratic Casimir $=18$ to give rise to a completely antisymmetric wave function [ए]] [2]].

To get an order of magnitude of the mass of the sexaquark, we can take the constituent masses (including the actractive chromoelectric interaction) from the baryons, $m_{u}=m_{d}=362 . \mathrm{MeV}$ and $m_{s}=539 \mathrm{MeV}$, and for the chromomagnetic interaction to keep into account that pair of quarks with the same flavor transform with respect to $S U(3)_{\times} S U(2)_{s}$ as the combination :

$$
\frac{1}{2}[\sqrt{3} \times(\overline{3}, 3)+(6,1)]
$$

while pair of quarks with different flavor as :

$$
\frac{\sqrt{2}}{4}\left[\sqrt{3} \times(\overline{3}, 1)+\sqrt{3} \times(6,3)+\frac{1}{2} \sqrt{3} \times(\overline{3}, 3)++\frac{1}{2}(6,1)\right]
$$

which implies a chromomagnetic contribution: $\frac{\left[5+22 k_{s}-3\left(k_{s}\right)^{2}\right]\left(M_{N}-M_{\Delta}\right)}{16}$, which added to the sum of the effective constituent masse, $4 m_{q}+2 m_{s}$, gives the value $2210 \mathrm{MeV}$ minor by a small amount than $2 m_{\Lambda}$.

Now we consider the possibility to have three diquarks $u d$, $u s$ and $d s$ transforming as the $(\overline{3}, 1, \overline{3})$ of $S U(3)_{c} \times S U(2)_{s} \times S U(3)_{f}$ as in the ingenious proposal by [䧃].

A similar possibility does not exist for three $u d$ pairs, since to combine into a color singlet gives rise to a totally antisymmetric wave function, not the right one for three bosons.

Scalar diquarks have been advocated by Maiani, Piccinini, Polosa and Riquier [ㅍ] to describe the lowest tetraquark nonet by combining them with there antiparticles.

The mass of a sexaquark built with three $(\overline{3}, 0)$ of $S U(3) c \times S U(2)_{s}$ is given by the sum of the masses of the three diquarks fixed by the description of the spectrum of the two scalar nonet tetraquarks given in the previous section.

In the case of the isoscalar with light costituents or with one strange costituent the sum of the masses of the two diquarks, which build the light and the strange tetraquarks is given by : 


$$
\begin{gathered}
2 m(u d)=4 m^{\prime \prime}<\alpha l|M| \alpha l>=4 m^{\prime \prime}+M_{N}-M_{\Delta} \\
m(u d)+m(u s)=3 m^{\prime \prime}+m_{s}^{\prime \prime}+<\alpha s|M| \alpha s>=3 m^{\prime \prime}+m_{s}^{\prime \prime}+\frac{1+k_{s}^{\prime \prime}}{2}\left[M_{N}-M_{\Delta}\right]
\end{gathered}
$$

we find for the light and strange scalar diquarks the masses $m(u d)=556.5 \mathrm{MeV}$ and $m(u s)=$ $m(d s)=663.2 \mathrm{MeV}$, respectively, which implies for the mass of the sexaquark

$$
M_{S}=m(u d)+m(u s)+m(d s)=4 m^{\prime \prime}+2 m_{s}^{\prime \prime}+\left(\frac{1}{2}+k_{s}^{\prime \prime}\right)\left[M_{N}-M_{\Delta}\right]+M_{\Sigma}-M_{Y^{*}}=1883 \mathrm{MeV}
$$

To show the reason how the value found for $M_{S}$ is more than $300 \mathrm{MeV}$ smaller than twice the mass of the $\Lambda$ we write an approximate mass formula, which takes into account of the small contribution of the chromomagnetic interaction to the masses of the heavier nonet slightly smaller than the sum of the constituent mases:

$$
M_{S}=2 M_{K(1430)}-\frac{1}{2} M_{f^{0}(1370)}+40 \mathrm{MeV}+\frac{1}{2}\left[M_{N}-M_{\Delta}\right]+M_{\Sigma}-M_{Y^{*}}=1876.5 \mathrm{MeV}
$$

which shows that the low value found for $M_{S}$ does not depend on the particular choice of the parameters. The first three terms in eq.(B.6) correspond to the constituent masses and the other terms to the contribution of the chromomagnetic interaction .

\section{Interactions of the sexaquark}

The hypothesis that $S$ is built by an antisymmetric in color and flavor combination of three scalar diquarks (us, ds,ud) has interesting consequences :

the reactions $K^{+}+S \rightarrow \Lambda+p$ and $\pi^{+}+S \rightarrow \Lambda+\Lambda$ advocated in [[]] to fastly reduce the number of $S$ 's formed, when the presence of strange quark was not so smaller than the one of the light quarks, may be less efficient of the reaction $\pi^{+}+d \rightarrow p+p$, which also reduces the number of constituents from 8 to 6 . In fact this reaction is induced by the large pion nucleon coupling $g_{N N \pi} \bar{N} \gamma_{5} \vec{\tau} \times \vec{\pi} N$, while the coupling of the initial pseudoscalar to change the flavor of a scalar diquark is forbidden from parity . Also a diquark cannot turn in a one with flavor already present for the antisymmetry in flavor. This properties play a role on the weak decays : in fact the transformation of a strange quark into a $u$ by a semileptonic interaction or into a $d$ by a non-leptonic are also forbidden for the antisymmetric property just mentioned.

\section{Conclusion}

After showing the role of the chromomagnetic interaction to the describe the spectrum of the lightest baryons and mesons, we relate the mass of the sexaquark built with three diquarks, $u d, u s$ and $d s$, trasforming as a $(\overline{3}, 0)$ of $S U(3)_{c} \times S U(2)_{s}$ in a symmetric $S$-wave combined in a singlet [四], to the masses of the two lowest scalar tetraquark nonets. The small value of the mass of the costituent strange quark in the diquarks leads to predict for the sexaquark a mass of $1883 \mathrm{MeV}$ just 
above the range advocated by [U] to propose that particle as a candidate for the dark mass.

The result found is a consequence of the low value found for the effective mass of the strange quark, $m_{s}^{\prime \prime}$, needed to descrive the spectrum of the two lowest scalar nonet tetraquarks and therefore needs a confirm .

The way, in which the constituents combine to build the sexaquark, gives rise to intriguing selection rules for its interactions and decays .

\section{Aknowledgements}

I thank Glennys Farrar for her encouragement and F. Guerra and P. Lipari for very useful discussions .

\section{References}

1) G. Farrar Phys. Rev. Lett. 120 (2018) 092102 and arXiv 1805.03723 [hep-ph]

2) E.W Kolb and M. Turner, Phys. Rev. D99 (2019) 063519 and arXiv 180906003 v1 [hep-ph] .

3) Talk by G. Farrar, IDM, 2018, Brown University .

4) C. Gross, A. Polosa, A Strumia, A. Urbano and W. Xue, Phys. Rev. D98 (2018) 063005.

5) M. Gaspero, Nucl. Phys. A562 (1975) 407.

6) R. Aaij et al.(LHCb Collaboration), Phys. Rev. Lett. 119 (2017) 112001.

7) M. Gell-Mann, CSTL-20 (1961),

S. Okubo Progr. Theor. Phys. 27 (5) (1962) 949 and 28 (1) (1962) 24

8) H. Fritzsch, M. Gell-Mann and H. Leutwyler, Phys. Lett. B47 (1973) 365 .

9) A. De Rújula, H. Georgi and S.L. Glashow, Phys. Rev. D12 (1975) 147.

10) E. G. Cazzoli, A. M. Cnops, P. L. Connolly, R. I. Louttit, M. S. Murtagh, P. A Palmer, N. P. Samios, T. T. Tao and H. H. Williams, Phys. Rev. Lett. 34, (1975) 1125.

11) H. Högasen and P. Sorba, Nucl. Phys. B145 (1978) 119.

12) M. de Combrugghes, H. Högaasen and P. Sorba, Nucl. Phys. B156 (1979) 347 .

13) F. Buccella, F. Nicolo' and A. Pugliese Lettere al Nuovo Cimento. 8 (1973) 244 .

14) S. Weinberg, Phys. Rev. 177 (1968) 2604.

15) F. Buccella, E. Celeghini, H. Kleinert, C. A. Savoy and E. Sorace Nuovo Cimento A69 (1970) 133 .

16)F. Buccella, E. Celeghini and C. A. Savoy Nuovo Cimento A70 (1972) 281 .

17) L. Maiani, F. Piccinini, A. D. Polosa and V. Riquer, Phys. Rev. Lett. 93 (2004) 212002.

18) G. 't Hooft, G. Isidori, L. Maiani, A. D. Polosa and V. Riquer, Phys. Lett. B662 (2008) 424 .

19) R. L. Jaffe, Phys. Rev. D17 (1978) 1444.

20) F. Buccella, Mod. Phys. Lett. A21 (2006) 831 
21) F. Buccella, H. Högaasen, J. M. Richard and P. Sorba Eur. Phys. J. C49 (2007) 743 .

22) F. Buccella and P. Sorba, Mod. Phys. Lett. A19 (2004) 1547.

23) M. Abud, F. Buccella, D. Falcone, G. Ricciardi and F. Tramontano, Adv. Stud. Theor. Phys. 2 (2008) 929 .

24) M. Abud, F. Buccella and F. Tramontano, Phys. Rev. D81 (2010) 0744018 . 